\title{
The Optofluidic Microscope - A High Resolution Microscope-on-A-Chip System
}

\author{
Xiquan Cui, Xin Heng, Lapman Lee and Changhuei Yang \\ Bioengineering and Electrical Engineering, California Institute of Technology, \\ Pasadena, CA, 91125
}

A complete microscope-a-chip system can form a vital component in a wide range of lab-on-a-chip (LOC) systems. Yet, the microscopy imaging needs associated with LOC systems are still generally fulfilled through the use of bulky conventional microscopes, which obviates the size advantage of LOC systems. Despite the need, a commercially viable approach for miniaturizing microscope system simply did not exist until recently. The difficulty is twofold. First, there isn't a cheap and efficient way to create small and precise optical lenses on chips easily. Second, the space requirements of conventional microscopy for light propagation and reshaping conflict with the size constraints of chip-based devices.

In this article, a complete on-chip high resolution OFM device is presented. The OFM method abandons the conventional microscope design and, instead, shares some similarities with direct shadow imaging method where the target object is directly placed on top of a CCD or CMOS imaging sensor chip [1]. In direct shadow imaging, the image resolution is no better than the sensor pixel size. As the typical sensor pixel size is around 10 microns, direct shadow imaging systems are unable to provide images that are comparable in resolution to those of conventional microscopes.

The OFM method $[2,3]$ overcomes this by flowing the target over a line of submicron holes that is etched onto a metal coated CMOS imaging sensor array; each hole transmits light to a unique pixel on the array. As the object passes over each hole along a microfluidic channel, it interrupts light transmission through the hole. The time varying transmission trace represents a line scan across the object. The slanted hole arrangement ensures that adjacent line scans overlap and the object is fully scanned. By stacking the line traces together appropriately, we can then construct an object's image with resolution given by the hole size.

We recently implemented a complete on-chip OFM system. In this device, the base sensor chip is a $640 \times 480$ CMOS imaging sensor with pixel size of $9.9 \mu \mathrm{m}$ (Fig. 2(a)). The window is removed to provide access to the chip die. We planarized the die with a $2 \mu \mathrm{m}$ thick layer of SU8 photoresist, and then plated it with a $300 \mathrm{~nm}$ thick layer of aluminum. Holes of diameter $500 \mathrm{~nm}$ were then fabricated on the metal layer via focused ion beam (FIB) machining; the hole spacing is $19.8 \mu \mathrm{m}$. Finally, a $15 \mu \mathrm{m}$ high and $50 \mu \mathrm{m}$ width channel in PDMS is placed onto of the hole array with the help of a mask aligner. The separation between adjacent holes across the channel is $250 \mathrm{~nm}$; this ensures that passing objects are fully scanned and sufficiently sampled. The chip is programmed in such way that it only outputs line scans from the pixel array directly beneath the hole array at a rate of 1500 frames per second. We are presently evaluating its capability to image blood cells and microorganisms.

To our knowledge, this system is the first complete on-chip lensless high resolution microscope system ever reported. The simplicity of the system design and the remarkable absence of precision optical elements make the OFM highly amendable to large scale fabrication and for inclusion in a wide range of LOC systems. Finally, its variants that have super-resolution, fluorescence sensitivity and/or phase imaging capability can be readily implemented. We shall briefly discuss our efforts in those directions during the proposed talk.

\footnotetext{
References

1. Lange, D., C.W. Storment, C.A. Conley and G.T.A. Kovacs, A microfluidic shadow imaging system for the study of the nematode Caenorhabditis elegans in space. Sensors And Actuators BChemical, 2005. 107(2): p. 904-914.

2. Heng, X., L.R. Baugh, D. Erickson, Z. Yaqoob, P.W. Sternberg, D. Psaltis and C. Yang, Optofluidic Microscope - A Method for Implementing A High Resolution Optical Microscope On A Chip. Lab On A Chip, 2006. 6: p. DOI: 10.1039/b604676b.
} 
3. Psaltis, D., R.S. Quake and C. Yang, Developing optofluidic technology through the fusion of microfluidics and optics. Nature, 2006. 442: p. 381.

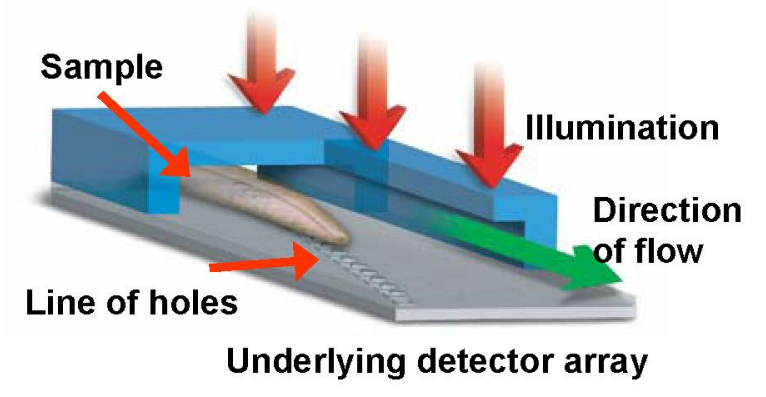

(a)

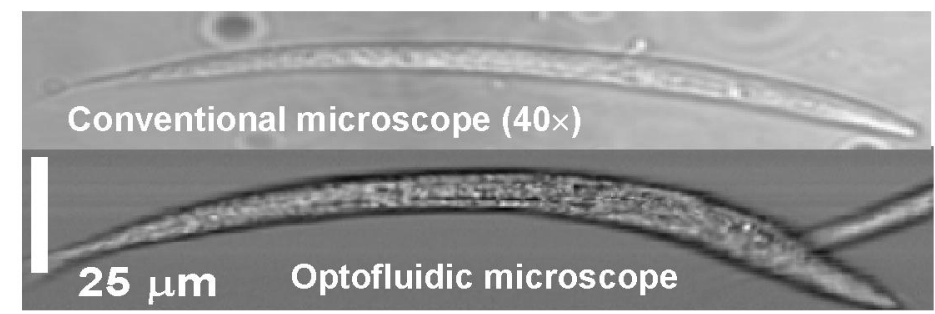

(b)

Figure 1. (a) The schematics of OFM, (b) Conventional microscope and OFM images of C. elegans.

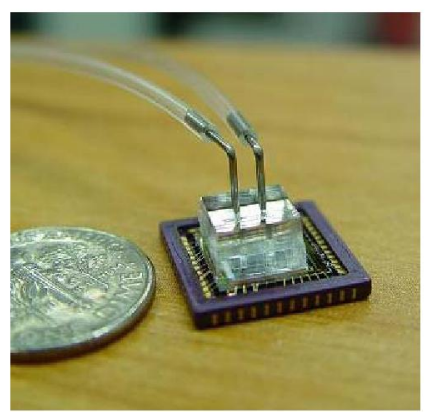

(a)

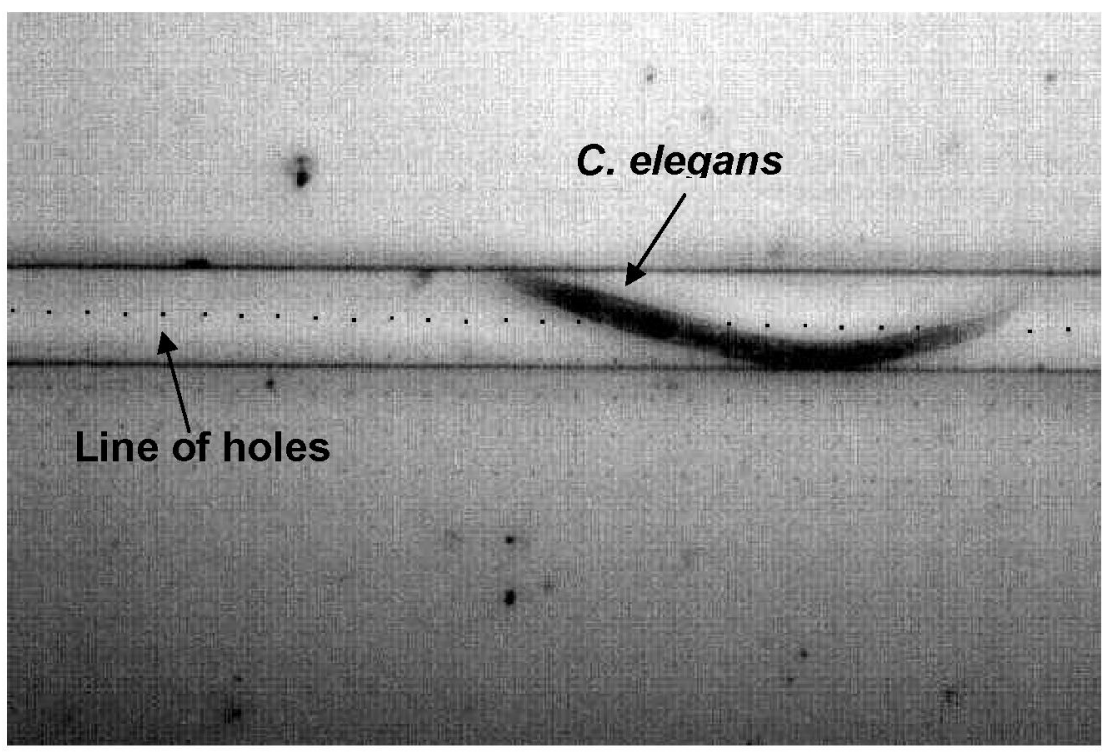

(b)

Figure 2. (a) The complete on-chip OFM device; (b) C. elegans flowing through the on-chip OFM device. The holes separation is 19.8 microns (or every other CMOS pixels) along the channel and $250 \mathrm{~nm}$ across the channel. The holes are $500 \mathrm{~nm}$. 